\title{
Thickness Optimization of Single Junction Quantum Well Solar Cell Using TCAD
}

\author{
Muhammad Johirul Islam ${ }^{1, a^{*}}$, Sanjina Mostafa ${ }^{2, b}$ \\ and Md. Iqbal Bahar Chowdhury $3, \mathrm{c}$ \\ ${ }^{1,2,3}$ Department of Electrical and Electronics Engineering, United International University, Dhaka, \\ Bangladesh \\ a*johir021@yahoo.com, bsanjina@ieee.org, cibchy@eee.uiu.ac.bd
}

\begin{abstract}
Keywords: Quantum well solar cells, Optimum thickness, Single-Junction cell, GaSb/GaAs, Silvaco TCAD.

Abstract. The efficiency increase by inserting quantum wells in a p-i-n solar cell has already been studied practically and theoretically over the years. Here we present a Multi-Quantum-well SingleJunction GaAs/GaSb solar cell which is simulated using Silvaco TCAD, where thicknesses of different layers have been varied to obtain the optimum thickness for maximum efficiency. Comparison is also presented for the same between the solar cells with and without the inclusion of quantum wells.
\end{abstract}

\section{Introduction}

The production of current by the photovoltaic effect using solar radiation was first discovered by Bequerel in 1839 [1]. And while the first solar cell was made by using selenium with a layer of gold, by New York inventor Charles Fritts in 1883 [2], physicists at Bell Laboratories found out that silicon was more efficient than selenium, and they introduced the first practical solar cell in the 1950s [3]. The research into solar cells has been in the rise since its discovery and decades have passed with the introduction of several types of solar cells using various types of materials. One of these is the thin-film solar cell which has the big advantage of being cheap in the expense of its efficiency. Several different enhancements can be made to the thin-film cells like point defect engineering [4], perfect light in coupling [5], thin-film solar cells fabricated by a two-step CdS deposition process [6] and thin film solar cells by cadmium alloying [7], amongst many others. The highest efficiency recorded from thin-film cells was around $29 \%$ with GaAs [8]. The problem with thin-film cells is that there is still alot of light which goes unabsorbed due to the solar cells' thickness but with the introduction of Quantum Wells (QWs) in between, some of this lost energy can be captured. In this paper we have used thin-film cells, together with QWs to analyse the effect of thickness. The thickness of a solar cell is an important consideration because it affects the price of the solar cell. We can have solar cells with very high efficiencies but if their price is very high, then it is not justified. Therefore, it is important to not only increase efficiency using quantum wells inside the intrinsic region but also to optimize the thickness of this quantum well solar cell by varying thickness of every layer in the QWSC. Insertion of multi-quantum wells in the i-layer of a standard solar cell increases conversion efficiency, that is, a multi quantum well solar cell is basically a p-i-n device incorporated by verythin layers of few nanometres of lower band gap inserted inside the intrinsic region, placed between the two doped layers, $\mathrm{n}$ and $\mathrm{p}$. This phenomenon was studied first by Barnham and their co-workers where they showed that quantum wells increase short circuit current [9]. In this research, we present a single junction $\mathrm{GaAs} / \mathrm{GaSb}$ quantum well solar cell where thickness has been varied for every layer and its efficiencies compared with the presence of wells and without wells. Our results show that only the i-layer needs to be big enough at around $1 \mathrm{um}$, while the rest of the layers needs to be around $1 \mathrm{~nm}$ for having the best efficiency/price ratio. This is true for both with and without wells, but implementation of quantum wells increases the efficiency beyond that of the conventional one. Previous research on thickness optimization has been done mainly on silicon solar cells but none so for quantum well solar cells $[10,11]$. Therefore, this analysis will be helpful to those who are looking to improve on the efficiency of quantum well solar cell while already having the optimum thickness. 


\section{Device Structure and Simulation}

The structure of quantum well solar cell (QWSC) can be a simple p-i-n solar cell which will have just 3 layers or the more advanced 6 layers for a more optimized solar cell. These are shown in Fig. 1, where we see the window come first, followed by emitter, i-layer, within which quantum wells are inserted, and then comes the base, BSF (back surface field) layer and finally, the substrate. The materials are also shown, mainly GaAs (bandgap is $1.42 \mathrm{eV}$ ) and AlGaAs (bandgap is $\approx 2.1 \mathrm{eV}$ ) are used here as absorber (in emitter, i-layer and base) and the window materials, respectively, not only to capture the maximum amount of light as possible but also to reduce the lattice mismatch and reduce losses. The wells are made of GaSb, which has a bandgap of $0.726 \mathrm{eV}$, and this has been selected so that recombination losses would be lowered. If a lower bandgap material had been used as quantum wells, then even though a higher wavelength photon could be absorbed, the recombination rate would be higher since the electron/hole had to cross a higher barrier.

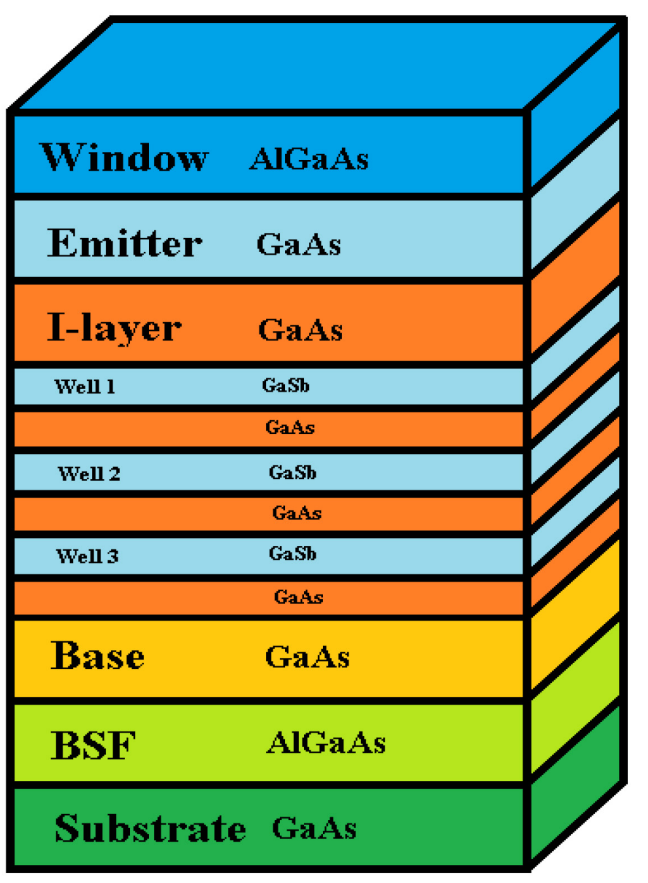

Fig. 1: Schematic of 6-layer QWSC

The whole structure is implemented in our work using SILVACO TCAD. The TCAD output for the said solar cell is shown in Fig 2. The materials, thicknesses, doping type and doping levels for each of the 6-layers are mentioned in Table 1. Barrier and well materials along with their thicknesses are also shown in the this table. 


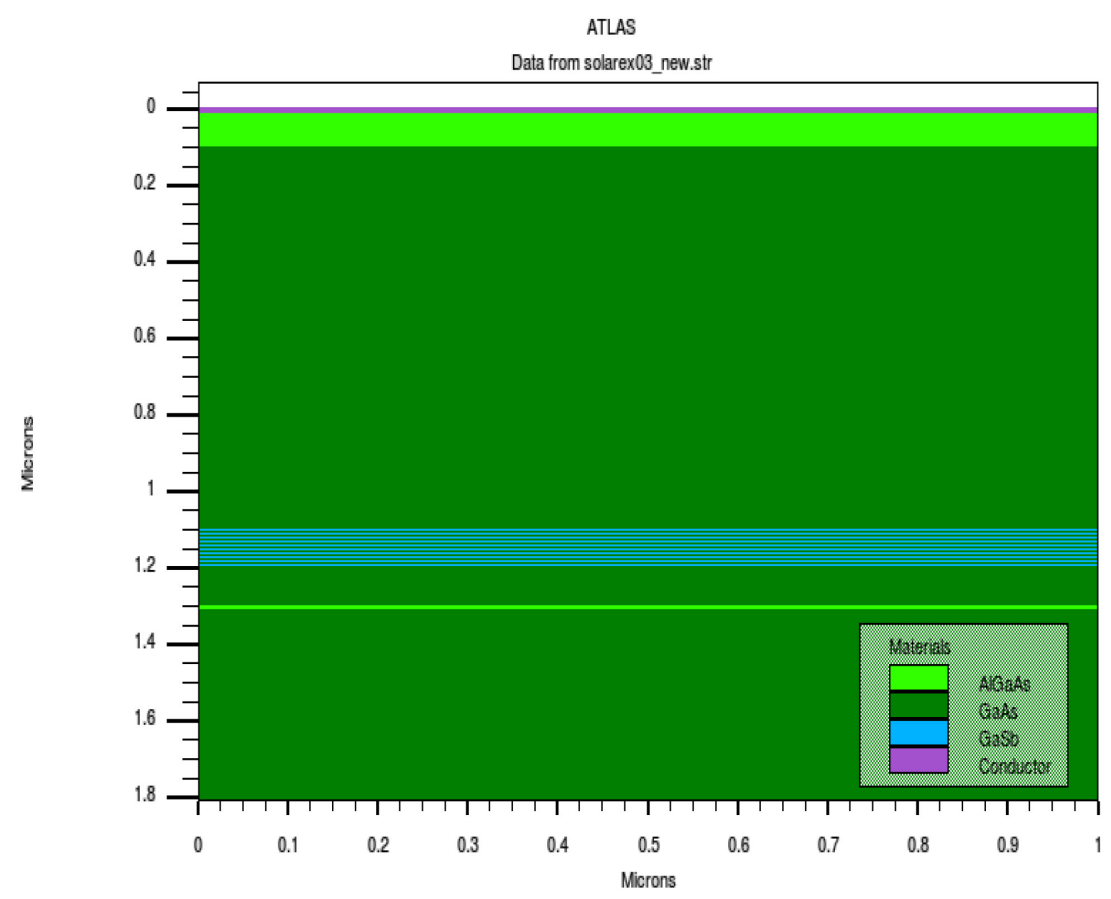

Fig. 2: Diagram of cell, 6 layers, 10 wells

Table 1: Layer materials, thicknesses, doping type and levels

\begin{tabular}{|c|c|c|c|c|}
\hline Layer & Material & Thickness $(\boldsymbol{\mu m})$ & $\begin{array}{c}\text { Doping } \\
\text { Type }\end{array}$ & $\begin{array}{c}\text { Doping } \\
\text { Level }\end{array}$ \\
\hline Window & Al0.9Ga0.1As & 0.1 & p & 1 e19 \\
\hline Emitter & GaAs & 0.1 & p & 2e18 \\
\hline i-layer & GaAs & 1.0 & i & - \\
\hline Base & GaAs & 0.1 & n & 2e18 \\
\hline BSF & Al0.9Ga0.1As & $\mathbf{0 . 0 1}$ & n & 5e18 \\
\hline Substrate & GaAs & $\mathbf{0 . 5}$ & n & 2e18 \\
\hline Barriers & GaAs & $\mathbf{0 . 0 0 5}$ & i & - \\
\hline Wells & GaSb & $\mathbf{0 . 0 0 5}$ & i & - \\
\hline
\end{tabular}

In order to perform quantum well based simulations, we have chosen k.p and qwell as the models.

\section{Results and Discussion}

We present here 6 graphs showing the effects of varying the thickness of the corresponding 6 layers in the quantum well solar cell. The six layers are window, emitter, i-layer, bsf and substrate. In Fig. 3, we see how changing the window thickness effects the efficiency. The window is a high bandgap semiconductor which only absorbs the high energy photons and allows the rest of the light to pass through (hence, the name window). In here we can see the general trend of efficiency decreasing with the thickness of the window. Since the window absorbs only high energy photons, only a small size of window is required to absorb these photons in which recombination effects are low. If we increase the thickness, then recombination effects have a greater effect and so efficiency decreases. The optimum thickness here is $1 \mathrm{~nm}$ which the first point of the graph 


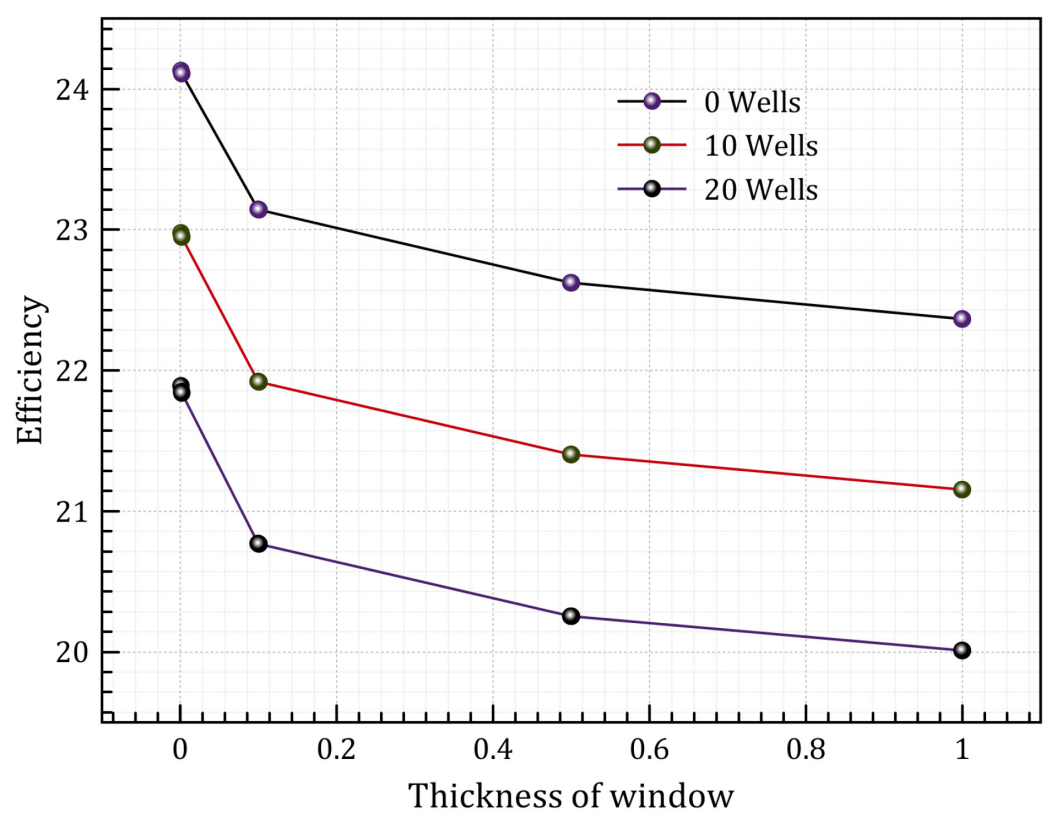

Fig. 3: Window thickness variation

The emitter also has a high bandgap to absorb the leftover high energy photons that have passed through the window. Here also, as shown in Fig. 4, increasing the emitter thickness decreases the efficiency due to increased recombination. Note that the sudden jump in efficiency from 0 to $1 \mathrm{~nm}$ shows that emitter has to be present in a solar cell.

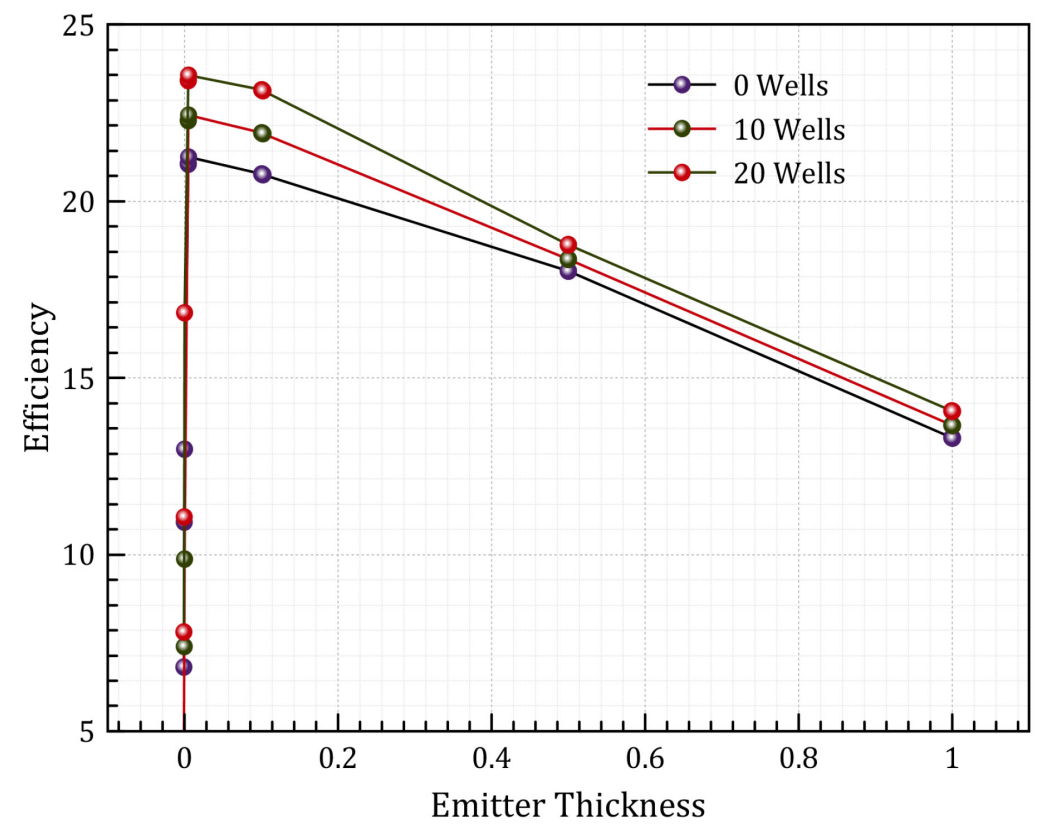

Fig. 4: Emitter thickness variation

The i-layer is introduced in between the p-n junction to increase the length of the region of electric field. (We know that electric field strength remains constant in i-region because there are no charges present, so having an i-region in the middle of $p-n$ junction will give a longer region of constant electric field than that observed in a p-n junction). We have seen from Fig. 5 that the efficiency increases as i-layer thickness increases. This is due to the fact that when thickness is increased, the region over which the electric field exists is increased. Hence, electrons/holes are in the electric field for longer length of region for which they are accelerated for a longer amount of time and hence, get less chance to recombine. This results in corresponding efficiency increase. 
Next, comes the base, which is the p-region. Here the lower energy photons are absorbed. As can be seen in Fig. 6, when there are 0 wells, the efficiency increases with increase in thickness, because more photons are absorbed and recombination is still less. But when wells are added, then thickness seems to have no effect, because the wells cause additional recombination which is balanced by the increase in absorption of photons due to increased thickness. Here also there is a sudden jump of efficiency from 0 to $1 \mathrm{~nm}$ which tells us that the base must be present in a solar cell.

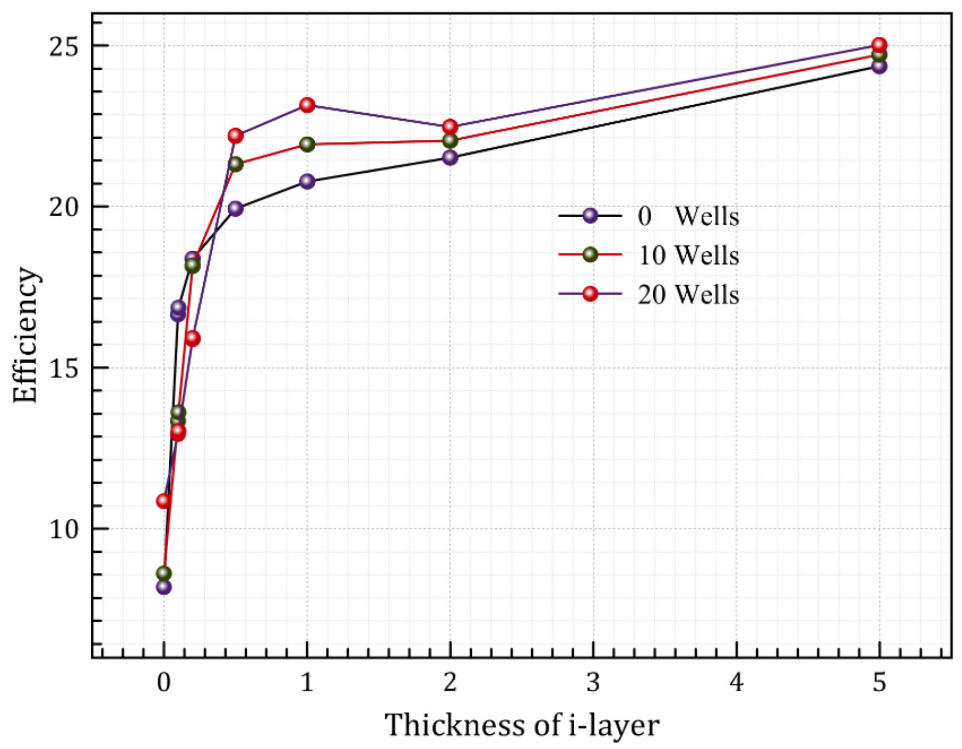

Fig. 5: I-layer thickness variation

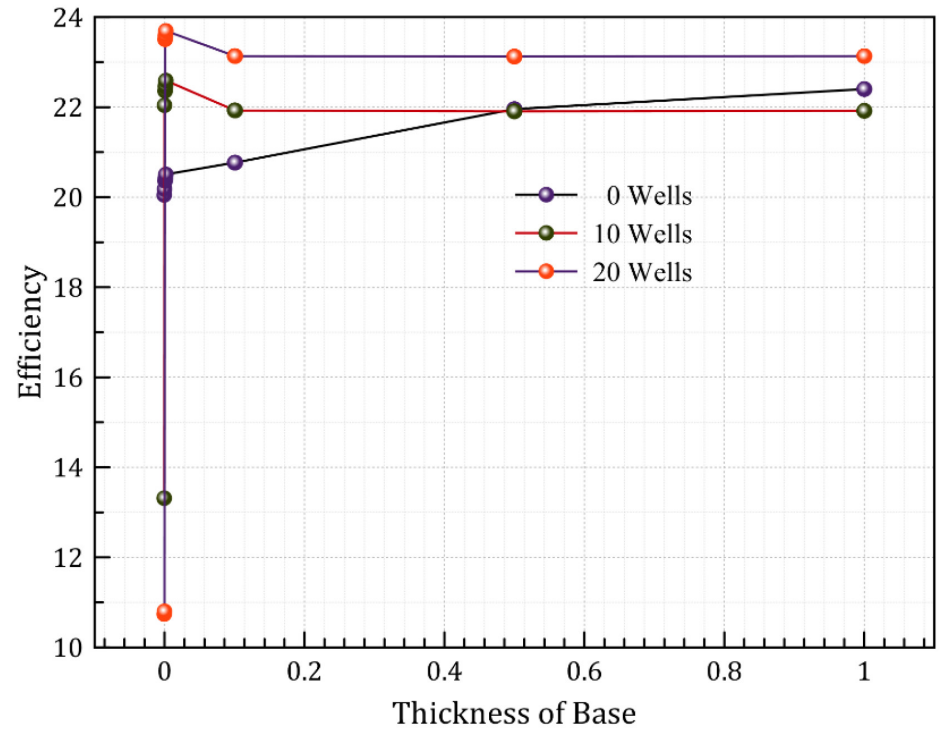

Fig. 6: Base thickness variation

The BSF (Back surface field) is also necessary since efficiency jump occurs from 0 to $1 \mathrm{~nm}$, as observed in Fig. 7. After that there is no increase because it has a high bandgap and high bandgap photons have already been absorbed before in window and emitter layers. The main aim here is to reduce surface recombination (which happens due to the surface not evenly cut) so a small thickness of it is sufficient.

The next and final layer would be the substrate and it is used to hold the solar cell, i.e. onto which the solar cell will be supported. The substrate is also necessary for solar cell otherwise there is a decrease in efficiency as shown in Fig. 8. However, increase in thickness has no effect since absorption and recombination increases at the same rate with the thickness. 


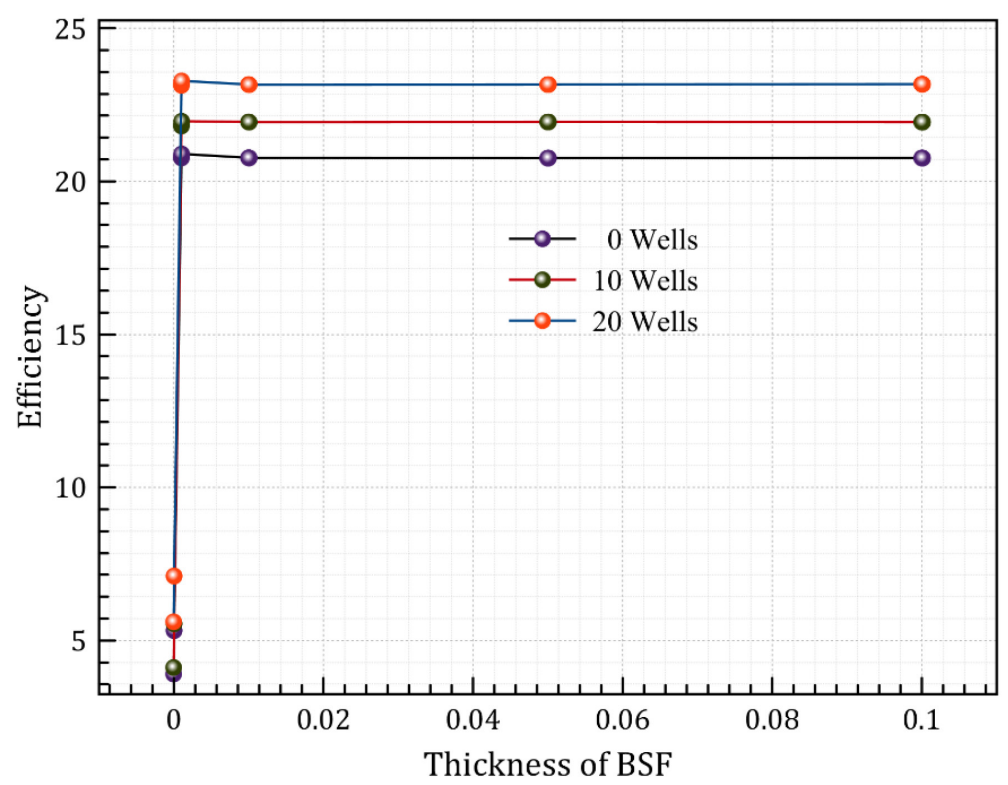

Fig. 7: Back surface field thickness variation

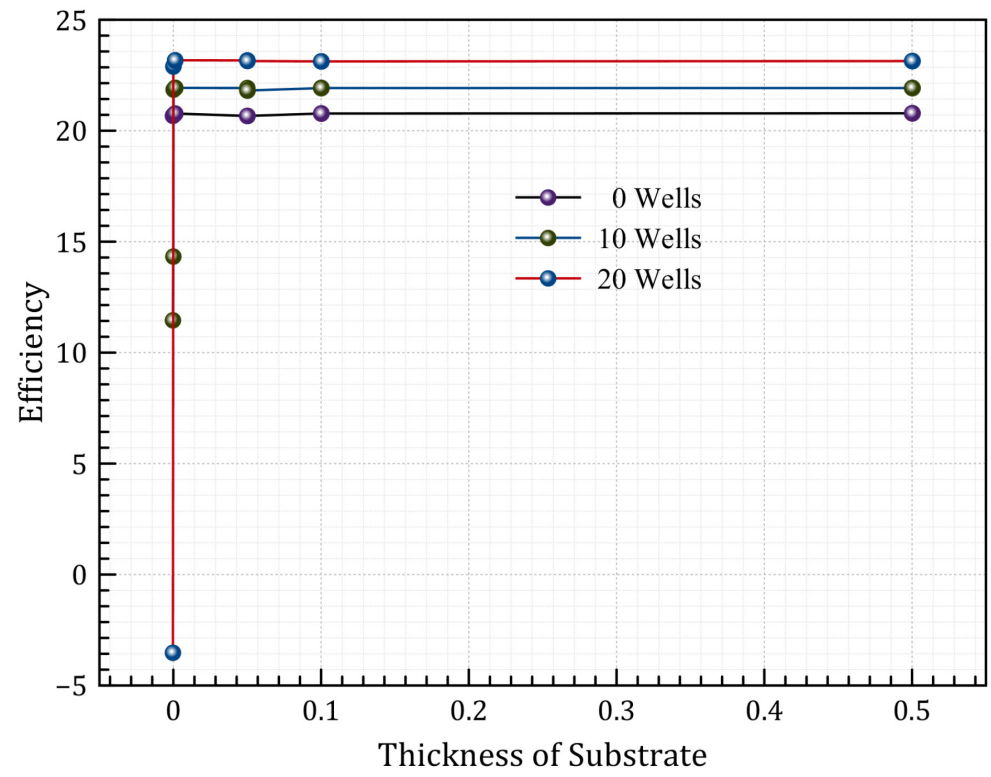

Fig. 8: Substrate thickness variation

Finally, the effect of number of quantum wells needs to be investigated. It has been observed from each of the figures of Fig. 3- 8 that number of quantum wells has significant effects on the efficiency of the solar cells. In all cases, when the number of quantum wells increases, the efficiency has been increased. When no quantum wells are present, the solar cell shows an efficiency close to $20 \%$; when 10 wells are inserted the efficiency is increased to about $22 \%$ and when 20 wells are employed, the efficiency moves to about $24 \%$. This clearly proves that insertion of quantum wells in the i-layer increases the absorption of low-energy photons and hence, increases the efficiency.

\section{Conclusion}

The simulation of single junction quantum well solar cell using GaSb wells have shown a clear increase in the efficiency. The simulations also show that the optimum thickness for i-layer of a single junction quantum well solar cell is around $1 \mu \mathrm{m}$, as after that the price increase due to thickness increase is not justified for the efficiency increase. Simulations also show that the rest of the layers are an essential part of the solar cell, and without any of these, the efficiency drops very low. The 
window and emitter layer thickness should not be greater than $0.1 \mu \mathrm{m}$; the base and BSF layers should be around $0.1 \mu \mathrm{m}$ thick, whereas, the substrate should be at most $0.5 \mu \mathrm{m}$ thick.

\section{References}

[1] A.E. Becquerel, C. R. Acad. Sci. 9 (1839),561.

[2] C. Fritts, On the Fritts selenium cell and batteries, Van Nostrand's Engineering Magazine, 32(1885), 388-395.

[3] D. M. Chapin, C. S. Fuller, and G. L. Pearson, A New Silicon p-n Junction Photocell for Converting Solar Radiation into Electrical Power, Journal of Applied Physics 25(1954), pp.676-677.

[4] Ji Sang Park, Sunghyun Kim, Zijuan Xie,Aron Walsh,Point defect engineering in thin-film solar cells, Nature Reviews Materials,3(2018),pp- 194-210

[5] Wayesh Qarony, Mohammad Ismail Hossain, Rahul Dewan, Stefan Fischer, V Benno MeyerRochow, Alberto Salleo, Dietmar Knipp, Yuen Hong Tsang, Approaching Perfect Light Incoupling in Perovskite and Silicon Thin Film Solar Cells by Moth Eye Surface Textures, Advanced Theory and Simulation,1(2018).

[6] Yaowei Wei, Daming Zhuang, Ming Zhao, Wenyu Zhang, Guoan Ren, Yixuan Wu, Rujun Sun, Qianming Gong, Leng Zhang, Shilu Zhan, Xiao Peng, Xunyan Lyu, Beyond 10\% efficient CZTSSe thin-film solar cells fabricated by a two-step CdS deposition process, olar Energy Materials and Solar Cells,180(2018), pp-19-24.

[7] Rujun Sun, Daming Zhuang, Ming Zhao, Qianming Gong, Mike Scarpulla, Yaowei Wei, Guoan Ren, Yixuan Wu, Beyond 11\% efficient Cu2ZnSn (Se, S) 4 thin film solar cells by cadmium alloying, Solar Energy Materials and Solar Cells,174(2018),pp- 494-498

[8] Martin A. Green, Yoshihiro Hishikawa, Ewan D. Dunlop, Dean H. Levi, Jochen Hohl-Ebinger, Masahiro Yoshita, Anita W.Y. Ho-Baillie, Solar cell efficiency tables (version 53), Progress in Photovoltaics: Research and Applications,27(2019),pp-3-12.

[9] K.W.J Barnham and G. Duggan, A new approach to high-efficiency multi-band-gap solar cells, Journal of Applied Physics,67(1990),pp-3490-3493.

[10] M. H. Abdullah, N. A. Rashied and M. Rusop, Layer thickness analysis of silicon solar cells, 2010 International Conference on Electronic Devices, Systems and Applications, 2010, pp-149153. 